\title{
铁电体自发极化的键模型
}

\section{一一钙钛矿、铇青铜矿铁电体自发极化强度 $p_{s}$ 的计算}

\author{
陈 孝 琛 \\ (中国科学院福建物质结构研究所)
}

本文提出了铁电体自发极化的键模型, 我们认为铁电体的自发极化是由于铁电体中键的 极性. 在高于居里点温度时, 铁电体由于结构对称性, 键的偶极矩互相抵消了; 在居里点以下 温度时, 由于铁电体的结构对称性发生了变化, 使键的偶极矩不能完全抵消, 所以出现了自发 极化 $p_{s} \neq 0$.

我们从固体中化学键的离子性出发,得到了铁电体中键偶极矩的计算式,并由键的偶极矩 计算了铁电体的自发极化强度 $p_{s}$. 我们计算了八种铁电体的自发极化强度, 并与前人的计算 结果以及实验值进行了比较, 与实验值符合较好.

\section{一、引 言}

自发极化强度是铁电体的一个基本物理量,它与铁电体的许多物理性质有关,如居里温度 $T_{c}$ 就与 $p_{s}$ 值有关; $p_{s}$ 值还与铁电体自发极化的微观机理密切联系, 如一维铁电体 $p_{s}$ 值最大, 二维、三维铁电体的 $p_{s}$ 值依次递减.

计算铁电体 $p_{s}$ 的比较简单的方法有线性关系 ${ }^{[1]}$ 和点电荷模型 ${ }^{[2]}$. 用这些方法计算铁电体 $p_{s}$, 其结果与实验相比较尚未能令人满意, 因此探索简单而又比较有效的计算方法是有意义 的. 我们提出的铁电体自发极化的键模型, 就是为了提供一种简单而比较有效的计算 $p_{s}$ 的方 法.

根据这个模型, 我们认为铁电体自发极化是由于键的极性, 即键的偶极矩, 铁电体在高于 居里点温度时, 由于晶体结构的膏对称性, 所以键偶极矩相互抵消, 铁电体 $p_{s}=0$; 当铁电体 经居里点向低温过渡时, 这时随着铁电体结构对称性的降低, 晶体结构发生畸变, 铁电体的键 偶极矩不能完全相互抵消, 此时铁电体就有了自发极化,也即 $p_{s} \neq 0$.

我们利用键模型计算了八种铁电体的自发极化强度,包括钙钛矿、铇青铜矿结构. $\mathrm{LiNbO}_{3} 、$ $\mathrm{KNbO}_{3} 、 \mathrm{LiT}_{2} \mathrm{O}_{3}$ 虽不属于钙钛矿,但晶体的基本结构单元也是氧八面体,所以也包括在内. 我 们把计算结果连同线性关系、点电荷模型的相应计算值与实验进行比较.

\section{二、计算方法与计算结果}

如前所述, 我们的键模型认为铁电体处在居里点以下温度时, 由于晶体结构发生畸变, 键 偶极矩不能完全抵消, 因而产生自发极化. 所以 $p_{s}$ 应是单位体积铁电体中键偶极矩的矢量

本文 1980 年 11 月 21 日收到。

第 3 期

科学通报 
和, 由于自发极化强度 $p_{s}$ 为铁电体单位体积中的偶极矩可得

$$
\vec{p}_{s}=\frac{1}{V} \sum_{\mu} G_{\mu} \vec{p}_{\mu},
$$

其中 $G_{\mu}$ 为键的结构因子, 为键与极化轴间的方向余弦; $V$ 为单胞体积; $\vec{p}_{\mu}$ 为键的偶极矩; $\mu$ 对单胞内键求和.

我们引人的计算键偶极矩的公式为

$$
p_{\mu}=2 e f_{\mu} d_{\mu},
$$

其中 $f_{\mu}$ 为键的离子性 (Ionicity), $d_{\mu}$ 为键长, $e$ 为电子电荷. $f_{\mu}$ 的定义 ${ }^{[3]}$ 如下:

$$
\begin{gathered}
f_{\mu}=C^{\mu^{2}} / E_{g}^{\mu^{2}}=C^{\mu^{2}} / C^{\mu^{2}}+E_{h}^{\mu^{2}}, \\
E_{h}^{\mu}=39.7 / d_{\mu}^{2.48}(\mathrm{eV}), \\
C^{\mu}=14.4 b^{\mu} \exp \left(-k_{s}^{\mu} \cdot r_{0}^{\mu}\right)\left[z_{\alpha}^{\mu} / r_{\alpha}^{\mu}-z_{\beta}^{\mu} / r_{\beta}^{\mu}\right]_{(\mathrm{eV})},
\end{gathered}
$$

$E_{g}^{\mu}$ 为键的平均能隙, $E_{h}^{\mu}$ 和 $C^{\mu}$ 为键平均能隙的对称和反对称部分, 以电子 - 伏特为单位. $z_{a}^{\mu}$, $z_{\beta}^{\mu}$ 和 $r_{\alpha}^{\mu}, r_{\beta}^{\mu}$ 分别为成键原子的价电子数和共价半径. $b^{\mu}$ 为屏蔽因子与平均配位数 $\bar{N}_{c}$ 平方 成正比 ${ }^{[4]}, \exp \left(-k_{s}^{\mu} \cdot r_{0}^{\mu}\right)$ 为 Thomas-Fermi 屏蔽因子, 其中

$$
\begin{gathered}
r_{0}^{\mu}=\frac{1}{2} d_{\mu}, \\
k_{s}^{\mu}=\left(4 k_{\mathrm{F}}^{\mu} / \pi a_{\mathrm{B}}\right)^{\frac{1}{2}},
\end{gathered}
$$

$k_{\mathrm{F}}^{\mu}$ 为 Formi 波矢, $a_{\mathrm{B}}$ 为 Bohr 半径.

$$
\left(k_{\mathrm{F}}^{\mu}\right)^{3}=3 \pi^{2} N_{e}^{\mu},
$$

$N_{c}^{\mu}$ 为键电子密度,

$n_{\mu}$ 为键价电子数, $v_{\mu}$ 为键体积,

$$
N_{e}^{\mu}=n_{\mu} / v_{\mu}
$$

$$
n_{\mu}=\left(z_{\alpha}^{\mu} / N_{c \alpha}^{\mu}\right)+\left(z_{\beta}^{\mu} / N_{c \beta}^{\mu}\right),
$$

$N_{c \alpha}^{\mu}, N_{c \beta}^{\mu}$ 为 $\mu$ 键的成键原子的配位数.

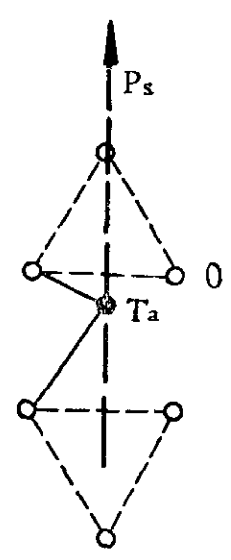

图 $1 \mathrm{Ta}$ 原子在氧八面体中 沿三重轴畸度, 形成 $\mathrm{T} a-\mathrm{O}$ 长、 短键

$$
v_{\mu}=\left(d_{\mu}\right)^{3} / \sum_{\nu}\left(d_{\nu}\right)^{3} N_{\nu}
$$

我们以计算 $\mathrm{LiTaO}_{3}$ 的 $p_{\text {: }}$ 为例说明我们的计算方法.

$\mathrm{LiTaO}_{3}$ 晶体的基本结构单元也是氧八面体，我们认为 $\mathrm{LiTaO}_{3}$ 的自发极化主要是氧八面体的贡献. 在居里点以下温度时, 氧八 面体的畸变对自发极化作出贡献. 在氧八面体中有 $\mathrm{O}-\mathrm{O}$ 键和 $\mathrm{Ta}-\mathrm{O}$ 键两种键, 其中 $\mathrm{O}-\mathrm{O}$ 键为纯共价性质 $\left(f_{\mu}=0\right.$, 因 $C^{\mu}=$ 0 ), 所以 $\mathrm{O}-\mathrm{O}$ 键的偶极矩为零, 不必计算, 只有六个 $\mathrm{Ta}-\mathrm{O}$ 键 偶极矩对自发极化有贡献. 在 $\mathrm{LiTaO}_{3}$ 晶体中, $\mathrm{Ta}$ 原子沿氧八面 体的三重对称轴畸变 ${ }^{[5]}$, 畸变后, $\mathrm{T} a-\mathrm{O}$ 的长键和短键由实线表 示(见图 1).

$$
\text { 中子衍射的结构数据 }{ }^{[6]} \text { : }
$$

六角单胞参数: $a_{H}=5.15428 \pm 0.00001 \AA, C_{H}=13.78351 \pm 0.00002 \AA$;

$\mathrm{Ta}-\mathrm{O}$ 的短键和长键: $d_{1}=1.908 \pm 0.003 \AA, d_{2}=2.073 \pm 0.003 \AA$;

$\mathrm{Ta}-\mathrm{O}$ 键的方向余弦: $n_{1}=0.507, n_{2}=0.647$. 
六角单胞体积 $V=3.17 \times 10^{-22} \mathrm{~cm}^{3}$. 每个单胞六个分子式. $f_{\mu}=0.756^{[7]}$.

按公式(1)、(2)可得 $\mathrm{LiTaO}_{3}$ 的自发极化强度

$$
p_{s}=\frac{1}{V} \sum_{\mu} G_{\mu} p_{\mu}=\frac{6}{V} 2 e f_{\mu} \cdot 3\left(n_{2} d_{2}-n_{1} d_{1}\right)=50.9 \mu \mathrm{C} / \mathrm{cm}^{2} .
$$

表 1 列出我们的计算结果, 连同线性关系、点电荷模型的相应计算值以及实验结果, 以便 比较.

表 1 八种铁电体的 $p_{s}\left(\mu C / \mathrm{cm}^{2}\right)$ 表*

\begin{tabular}{c|c|c|c|c}
\hline 铁电体 & 实验 值 & 点电荷 & 线性关系 & 键模型 \\
\hline $\mathrm{LiNbO}_{3}$ & 71 & 59 & 69 & 69.9 \\
$\mathrm{KNbO}_{3}$ & 30 & 23 & 41 & 35.7 \\
$\mathrm{Li}^{\mathrm{T} \mathrm{CaO}_{3}}$ & 50 & 49 & 51 & 50.9 \\
$\mathrm{BaTiO}_{3}$ & 26 & 33 & 30.3 \\
$\mathrm{PbTiO}_{3}$ & 75 & 44 & 64 & 70.9 \\
$\mathrm{BNN}$ & 40 & 26 & 53 & 40.3 \\
$\mathrm{SBN}$ & $25 * *$ & 9 & 27 & 23.6 \\
$\mathrm{KLN}$ & - & 20 & 42 & 34.0 \\
\hline
\end{tabular}

* 表中所列 $p_{s}$ 佳见文献[1]、[2]. ** 见文献[8].

\section{三、讨 论}

（1）从理论计算与实验值比较来看, 我们一般比线性关系和点电荷模型好. 点电荷模型 结果与实验值相差较大, 这与形式电荷有关.

（2）从我们的 $p_{s}$ 计算公式(1)、(2) 来看,线性关系只是我们的一种特殊情况, 因为对于上 述铁电体如果 $f_{\mu}$ 和 $V$ 为常数, 则

$$
p_{s}=\frac{1}{V} \sum_{\mu} G_{\mu} p_{\mu}=\frac{2 e f_{\mu}}{V} \sum_{\mu} G_{\mu} d_{\mu} \propto \Delta z,
$$

即 $p$ 。正比于配位多面体中心原子的位移 $\Delta z$.

（3）我们计算方法的出发点是固体中化学键的极性, 我们从键的偶极矩计算铁电体自发 极化强度. 在我们的计算公式中, 没有任何可调整参数.

（4）由公式(2)可知, 键偶极矩与 $f_{\mu}$ 成正比, 因此纯共价晶体 $\left(f_{\mu}=0\right)$ 键偶极矩 $p_{\mu}=0$, 所以不可能有自发极化, 即不可能是铁电体, 也不可能是热释电或压电材料.

\section{参考 文 献}

[1] Abrahams, S. C., Kurtz, S. K. \& Jamieson, P. B., Phys. Rev., $172(1968), 551$.

[2] Abrahams, S. C., \& Keve, E. T., Ferroelectrics, 2(1971), 129.

[3] Phillips, J. C., Rev. Mod. Phys., 42(1970), 317.

14] Levine, B. F., J. Chem. Phys., 59(1973), 1463.

[5] Abrahams, S. C. \& Bernstein, J. L., J. Phys. Chem. Solids, 28(1967), 1685.

[6] Abrahams, S. C., J. Phys. Chem. Solids, 28(1967), 1693.

[ 7 ] Levine, B. F., Phys. Rev. BIO., 1974, 1655.

[8] Lines, M. E. \& Glass, A. M., Principles and Applications of Ferroelectrics and Related Materials, Clarendon Press, Oxford, 1977, 500. 